\title{
Lie derivatives, forms, densities, and integration
}

\author{
John L. Friedman
}

Department of Physics, University of Wisconsin-Milwaukee 


\section{Lie derivatives}

Lie derivatives arise naturally in the context of fluid flow and are a tool that can simplify calculations and aid one's understanding of relativistic fluids.

Begin, for simplicity, in a Newtonian context, with a stationary fluid flow with 3 -velocity $\mathbf{v}(\mathbf{r})$. A function $f$ is said to be dragged along by the fluid flow, or Liederived by the vector field $\mathbf{v}$ that generates the flow, if the value of $f$ is constant on a fluid element, that is, constant along a fluid trajectory $\mathbf{r}(t)$ :

$$
\frac{d}{d t} f[\mathbf{r}(t)]=\mathbf{v} \cdot \nabla f=0 .
$$

The Lie derivative of a function $f$, defined by

$$
\mathcal{L}_{\mathbf{v}} f=\mathbf{v} \cdot \nabla f
$$

is the directional derivative of $f$ along $\mathbf{v}$, the rate of change of $f$ measured by a comoving observer.

Consider next a vector that joins two nearby fluid elements, two points $\mathbf{r}(t)$ and $\overline{\mathbf{r}}(t)$ that move with the fluid: Call the connecting vector $\lambda \mathbf{w}$, so that for small $\lambda$ the fluid elements are nearby: $\lambda \mathbf{w}=\overline{\mathbf{r}}(t)-\mathbf{r}(t)$. Then $\lambda \mathbf{w}$ is said to be dragged along by the fluid flow, as shown in Fig. (1). In the figure, the endpoints of $\mathbf{r}\left(t_{i}\right)$ and $\overline{\mathbf{r}}\left(t_{i}\right)$ are labeled $\mathrm{r}_{\mathrm{i}}$ and $\overline{\mathrm{r}}_{\mathrm{i}}$.

A vector field $\mathbf{w}$ is Lie-derived by $\mathbf{v}$ if, for small $\lambda, \lambda \mathbf{w}$ is dragged along by the fluid flow. To make this precise, we are requiring that the equation

$$
\mathbf{r}(t)+\lambda \mathbf{w}(\mathbf{r}(t))=\overline{\mathbf{r}}(t)
$$

be satisfied to $O(\lambda)$. Taking the derivative of both sides of the equation with respect to $t$ at $t=0$, we have

$$
\begin{aligned}
\mathbf{v}(\mathbf{r})+\lambda \mathbf{v} \cdot \nabla \mathbf{w}(\mathbf{r}) & =\mathbf{v}(\overline{\mathbf{r}})=\mathbf{v}[\mathbf{r}+\lambda \mathbf{w}(\mathbf{r})] \\
& =\mathbf{v}(\mathbf{r})+\lambda \mathbf{w} \cdot \nabla \mathbf{v}(\mathbf{r})+O\left(\lambda^{2}\right)
\end{aligned}
$$

which holds if and only if

$$
[\mathbf{v}, \mathbf{w}] \equiv \mathbf{v} \cdot \nabla \mathbf{w}-\mathbf{w} \cdot \nabla \mathbf{v}=0 .
$$

The commutator $[\mathbf{v}, \mathbf{w}]$ is the Lie derivative of $\mathbf{w}$ with respect to $\mathbf{v}$, written

$$
\mathcal{L}_{\mathbf{v}} \mathbf{w}=[\mathbf{v}, \mathbf{w}]
$$




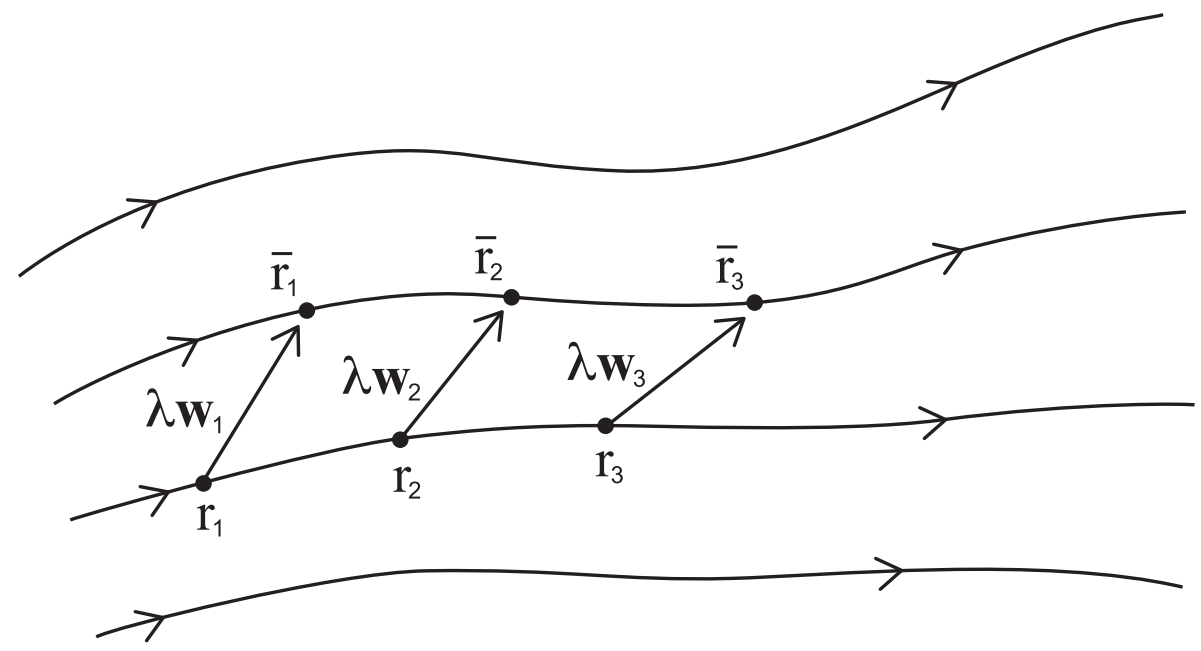

Figure 1: Two nearby fluid elements move along the flow lines, their successive positions labeled $r_{i}$ and $\bar{r}_{i}$. A vector field $\lambda w$ is said to be dragged along by the flow when, as shown here, it connects successive positions of two nearby fluid elements.

Then $\mathbf{w}$ is Lie-derived by $\mathbf{v}$ when $\mathcal{L}_{\mathbf{v}} \mathbf{w}=\mathbf{0}$. The Lie derivative $\mathcal{L}_{\mathbf{v}} \mathbf{w}$ compares the change in the vector field $\mathbf{w}$ in the direction of $\mathbf{v}$ to the change that would occur if $\mathbf{w}$ were dragged along by the flow generated by $\mathbf{v}$. In a curved spacetime the Lie derivative of a function $f$ is again its directional derivative,

$$
\mathcal{L}_{\mathbf{u}} f=u^{\alpha} \nabla_{\alpha} f .
$$

If $u^{\alpha}$ is the 4-velocity of a fluid, generating the fluid trajectories in spacetime, $\mathcal{L}_{\mathbf{u}} f$ is commonly termed the convective derivative of $f$. The Newtonian limit of $u^{\alpha}$ is the 4-vector $\partial_{t}+\mathbf{v}$, and $\mathcal{L}_{\mathbf{u}} f$ has as its limit the Newtonian convective derivative $\left(\partial_{t}+\mathbf{v} \cdot \nabla\right) f$, again the rate of change of $f$ measured by a comoving observer. (Now the flow is arbitrary, not the stationary flow of our earlier Newtonian discussion.)

A connecting vector is naturally a contravariant vector, the tangent to a curve joining nearby points in a flow; and in a curved spacetime, the Lie derivative of a contravariant vector field is again defined by Eq. (6),

$$
\mathcal{L}_{\mathbf{u}} w^{\alpha}=u^{\beta} \nabla_{\beta} w^{\alpha}-w^{\beta} \nabla_{\beta} u^{\alpha} .
$$


We have used a fluid flow generated by a 4-velocity $u^{\alpha}$ to motivate a definition of Lie derivative; the definition, of course, is the same in any dimension and for any vector fields:

$$
\mathcal{L}_{\mathbf{v}} w^{a}=v^{b} \nabla_{b} w^{a}-w^{b} \nabla_{b} v^{a} .
$$

Although the covariant derivative operator $\nabla$ appears in the above expression, it is in fact independent of the choice of derivative operator. This is immediate from the symmetry $\Gamma_{j k}^{i}=\Gamma_{(j k)}^{i}$, which implies that the components have in any chart the form

$$
\mathcal{L}_{\mathbf{v}} w^{i}=v^{j} \partial_{j} w^{i}-w^{j} \partial_{j} v^{i}
$$

One can extend the definition of Lie derivative to arbitrary tensors by requiring that, for a product, it act as a derivative, satisfying the Leibnitz rule: Thus, for any covector $\sigma_{a}$, and any vector $e^{a}$, one requires

$$
\mathcal{L}_{\mathbf{v}}\left(\sigma_{a} e^{a}\right)=\left(\mathcal{L}_{\mathbf{v}} \sigma_{a}\right) e^{a}+\sigma_{a} \mathcal{L}_{v} e^{a} .
$$

Because the component $\sigma_{a} e^{a}$ is a scalar, its Lie derivative is the directional derivative $v \cdot \nabla(\sigma \cdot e)=v \cdot \partial(\sigma \cdot e)$. From Eqs. (11) and (9), we have

$$
\begin{aligned}
\left(\mathcal{L}_{\mathbf{v}} \sigma_{a}\right) e^{a}+\sigma_{a}\left(v^{b} \nabla_{b} e^{a}-e^{b} \nabla_{b} v^{a}\right) & =v^{b} \nabla_{b}\left(\sigma_{a} e^{a}\right)=\left(v^{b} \nabla_{b} \sigma_{a}\right) e^{a}+\sigma_{a} v^{b} \nabla_{b} e^{a} \\
e^{a} \mathcal{L}_{\mathbf{v}} \sigma_{a} & =e^{a} v^{b} \nabla_{b} \sigma_{a}+e^{a} \sigma_{b} \nabla_{a} v^{b}
\end{aligned}
$$

Because the value of $e^{a}$ at any one point along the trajectory is arbitrary, we have

$$
\mathcal{L}_{\mathbf{v}} \sigma_{a}=v^{b} \nabla_{b} \sigma_{a}+\sigma_{b} \nabla_{a} v^{b}
$$

Again it is easy to check that the definition (12) is independent of the choice of derivative operator, that the components in any chart are given by

$$
\mathcal{L}_{\mathbf{v}} \sigma_{i}=v^{j} \partial_{j} \sigma_{i}+\sigma_{j} \partial_{i} v^{j}
$$

Finally, the Lie derivative of an arbitrary tensor $T^{a_{1} \cdots a_{m}} b_{1} \cdots b_{n}$ again follows from the Leibnitz rule applied to $\mathcal{L}_{\mathbf{v}}\left(T^{a \cdots b}{ }_{c \cdots d} e_{i}^{a} \cdots e_{j}^{b} \omega_{c}^{k} \cdots \omega_{d}^{l}\right)$, for arbitrary vectors $e_{1}^{a}, \ldots, e_{n}^{a}$ and covectors $\omega_{a}^{1}, \ldots, \omega_{a}^{m}$ :

$$
\begin{aligned}
\mathcal{L}_{\mathbf{v}} T_{b_{1} \cdots b_{n}}^{a_{1} \cdots a_{m}}= & v^{c} \nabla_{c} T^{a_{1} \cdots a_{m}}{ }_{b_{1} \cdots b_{n}} \\
& -T^{c \cdots a_{m}}{ }_{b_{1} \cdots b_{n}} \nabla_{c} v^{a_{1}}-\cdots-T^{a_{1} \cdots c}{ }_{b_{1} \cdots b_{n}} \nabla_{c} v^{a_{m}} \\
& +T^{a_{1} \cdots a_{m}}{ }_{c \cdots b_{n}} \nabla_{b_{1}} v^{c}+\cdots+T^{a_{1} \cdots a_{m}}{ }_{b_{1} \cdots c} \nabla_{b_{n}} v^{c},
\end{aligned}
$$


independent of the derivative operator, and with components in a chart given by

$$
\begin{aligned}
\mathcal{L}_{\mathbf{v}} T^{i_{1} \cdots i_{m}}{ }_{j_{1} \cdots j_{n}}= & v^{k} \partial_{k} T^{i_{1} \cdots i_{m}}{ }_{j_{1} \cdots j_{n}} \\
& -T^{k \cdots i_{m}}{ }_{j_{1} \cdots j_{n}} \partial_{k} v^{i_{1}}-\cdots-T^{i_{1} \cdots k}{ }_{j_{1} \cdots j_{n}} \partial_{k} u^{i_{m}} \\
& +T^{i_{1} \cdots i_{m}}{ }_{k \cdots j_{n}} \partial_{j_{1}} v^{k}+\cdots+T^{i_{1} \cdots i_{m}}{ }_{j_{1} \cdots k} \partial_{j_{n}} v^{k} .
\end{aligned}
$$

Action of diffeos ${ }^{1}$ and relation to Lie derivatives. We began by using the flow generated by a velocity field $\mathbf{v}$ to motivate the definition (6) of Lie derivative of a vector field $\mathbf{w}$. It is useful to see formally the way in which any vector field generates a flow and to use that flow to give a geometrical definition of Lie derivative. Note first that the trajectory (worldline) of a fluid element is an integral curve of the vector field $u^{\alpha}$, where:

Definition. An integral curve $c(\lambda)$ of a vector field $\xi^{a}$ is a curve whose tangent vector at each point $P=c\left(\lambda_{0}\right)$ is $\xi^{a}(P)$.

In a chart $\left\{x^{i}\right\}$, the tangent $\xi^{i}$ to an curve $c(\lambda)$ has components $\frac{d}{d \lambda} c^{i}(\lambda)$; and the statement that $c(\lambda)$ is an integral curve has the form $\frac{d}{d \lambda} c^{i}(\lambda)=\xi^{i}[c(\lambda)]$.

Proposition. Any smooth vector field $\xi^{a}$ in an n-dimensional manifold $M$ has a family of integral curves, one through each point of $M .^{2}$

Example 1: As noted, the velocity field $u^{\alpha}$ of a fluid has as its integral curves the fluid trajectories parameterized by proper time. The 3 -dimensional vector field $\mathbf{v}$ of a stationary Newtonian flow has as its integral curves the flow lines, parameterized by Newtonian time.

Example 2: The vector field $\boldsymbol{\partial}_{\phi}=x \boldsymbol{\partial}_{y}-y \boldsymbol{\partial}_{x}$ has as integral curves the lines of constant $t, r, \theta$,

$$
\lambda \rightarrow(t, r, \theta, \phi+\lambda)
$$

Note that when a vector field vanishes at $P$ (e.g., $\partial_{\phi}$ vanishes on the symmetry axis $x=y=0$ ) the integral curve simply stays at $P: c(\lambda)=P$.

\footnotetext{
${ }^{1}$ A smooth, 1-1 map of a manifold onto itself or onto another manifold is called a diffeo (or diffeomorphism).

${ }^{2}$ This result is equivalent to the existence theorem for solutions to ordinary differential equations, proved, for example, in Coddington and Levinson [1].
} 
We can view the 4-dimensional flow of a fluid as a family of smooth maps of the fluid to itself in the following way: In a given proper time $\tau$ each point $P$ in the fluid moves along the fluid trajectory through $P$ from $c(0)=P$ to the point $c(\tau)$. As in the case of the 4-velocity, we can use the integral curves of any vector field to define a family $\psi_{\lambda}$ of diffeos of a manifold to itself (for a star, the fluid has a boundary, and the map $\psi_{\tau}$ is from the support of the fluid to itself):

For each point $P$ let $c(\lambda)$ be the integral curve of $\xi^{a}$ for which $P=c(0)$. For a fixed value $\lambda$, define the map $\psi_{\lambda}$ by

$$
\psi_{\lambda}(P)=c(\lambda)
$$

That is, $\psi_{\lambda}$ maps each point $P$ to the point a parameter distance $\lambda$ from $P$ along the integral curve through $P$. The vector field $\xi^{a}$ is said to generate the family $\psi_{\lambda}$ of diffeos. In a chart $\left\{x^{i}\right\}$, we have

$$
\xi^{i}(x)=\left.\frac{d}{d \lambda} \psi_{\lambda}^{i}(x)\right|_{\lambda=0} .
$$

Example: The vector field $\boldsymbol{\partial}_{\phi}$ generates the family of diffeos

$$
(t, r, \theta, \phi) \rightarrow(t, r, \theta, \phi+\lambda),
$$

rotations by $\lambda$ in the $x-y$ plane about the axis where $\partial_{\phi}$ vanishes.

We can now repeat for manifolds the relation with which we began this section, between the flow - the diffeos - generated by a vector field and the Lie derivative. We again need the action of a diffeo $\psi$ on a tensor $T$

The definition of $\psi T$ is closely tied to our intuitive understanding of a fluid flow. As in the flat-space discussion at the beginning of this section, we start with functions and vectors. A function $f$ of a fluid is conserved if the fluid drags the function along with it. Define the dragged-along function $\psi f$ by requiring that $\psi f[\psi(P)]=f(P)$, or

$$
\psi f(P):=f\left[\psi^{-1}(P)\right] .
$$

A function $f$ on a fluid is Lie-derived by the fluid flow if

$$
\frac{d}{d \tau} \psi_{\tau} f \equiv-\mathcal{L}_{\mathbf{u}} f=0
$$

where $\psi_{\tau}$ is again the family of diffeos generated by the fluid velocity $u^{\alpha}$. Then, for any smooth vector field $\xi^{a}$ and function $f$ on a manifold $M$, one can define the Lie derivative $\mathcal{L}_{\xi} f$ by

$$
\mathcal{L}_{\xi} f=-\frac{d}{d \lambda} \psi_{\lambda} f
$$


where $\psi_{\lambda}$ is the family of diffeos generated by $\xi^{a}$.

To extend the action of diffeos to vector fields, we consider a curve $c(s)$ joining two fluid elements, and let $w^{\alpha}$ be its tangent vector. For small $s, s w^{\alpha}$ can be regarded as a connecting vector joining fluid elements a distance $s$ apart. For a fixed proper time $\tau$, write $\psi \equiv \psi_{\tau}$. The fluid flow maps the curve $c(s)$ to a curve $\psi[c(s)]$, dragged along a proper time $\tau$ with the fluid flow. The tangent vector $w^{\alpha}$ to $c$ at a point $P$ is in this way dragged along to a vector $\psi w^{\alpha}$ tangent to $\psi \circ c$ at $\psi(P)$, a proper distance $\tau$ from $P$. Its components are

$$
\left.\psi w^{\mu}\right|_{\psi(P)}=\left.\frac{d}{d s} \psi^{\mu}[c(s)]\right|_{s=0}=\left.\partial_{\nu} \psi^{\mu} \frac{d c^{\nu}}{d s}\right|_{s=0}=\partial_{\nu} \psi^{\mu} w^{\nu}(P) .
$$

Equivalently, $\psi w^{\mu}(P)=\partial_{\nu} \psi^{\mu} w^{\nu}\left[\psi^{-1}(P)\right]$.

More generally, any diffeo $\psi$ of a manifold $M$ to itself drags a vector field $w^{a}$ to a vector field $\psi w^{a}$, with

$$
\psi w^{i}(P)=\partial_{j} \psi^{i} w^{j}\left[\psi^{-1}(P)\right] .
$$

A vector field $w^{a}$ is Lie-derived by a vector field $\xi^{a}$ if the family of diffeos $\psi_{\lambda}$ generated by $\xi^{a}$ leave $w^{a}$ unchanged, if $\psi_{\lambda} w^{a}=w^{a}$; and the Lie derivative is again given by

$$
\mathcal{L}_{\mathbf{v}} w^{a}=-\frac{d}{d \lambda} \psi_{\lambda} w^{a} .
$$

To check that this agrees with our earlier definition, we use Eq. (17) and $\psi_{0}=$ identity, writing

$$
\begin{aligned}
\left.\frac{d}{d \lambda}\left(\left.\psi_{\lambda} w^{i}\right|_{P}\right)\right|_{\lambda=0} & =\frac{d}{d \lambda}\left[\frac{\partial \psi_{\lambda}^{i}}{\partial x^{j}} w^{j}\left(\psi_{\lambda}^{-1}(P)\right)\right]_{\lambda=0} \\
& =\frac{\partial}{\partial x^{j}}\left(\frac{d \psi_{\lambda}^{i}}{d \lambda}\right)_{\lambda=0} w^{j}(P)+\left.\frac{\partial \psi_{\lambda}^{i}}{\partial x^{j}}\right|_{\lambda=0} \frac{d}{d \lambda} w^{j}\left(\psi_{\lambda}^{-1}(P)\right) .
\end{aligned}
$$

Using

$$
\frac{d}{d \lambda} w^{i}\left(\psi_{\lambda}^{-1}(P)\right)=\frac{\partial w^{i}}{\partial x^{j}} \frac{d \psi_{-\lambda}^{j}(P)}{d \lambda}=-\xi^{j} \partial_{j} w^{i} \quad \text { and }\left.\quad \frac{\partial \psi_{\lambda}^{i}}{\partial x^{j}}\right|_{\lambda=0}=\delta_{j}^{i},
$$

we have

$$
\begin{aligned}
\left.\frac{d}{d \lambda} \psi_{\lambda} w^{i}\right|_{\lambda=0} & =\partial_{j} \xi^{i} w^{j}-\xi^{j} \partial_{j} w^{i} \\
& =-\mathcal{L}_{\xi} w^{i} .
\end{aligned}
$$


The action of a diffeo on a covector $\sigma_{a}$ can be found by writing it, at a point $P$, as the gradient of a function. The covector field $\nabla_{a} f$ is in this way dragged to a covector field $\psi \nabla_{a} f=\nabla_{a}(\psi f)=\nabla_{a}\left[f \circ \psi^{-1}\right]$, with components given by

$$
\left.\nabla_{i}(\psi f)\right|_{P}=\left.\partial_{i}\left(f \circ \psi^{-1}\right)\right|_{P}=\left.\partial_{j} f\right|_{\psi^{-1}(P)} \partial_{i} \psi^{-1 j}
$$

Then $\psi$ drags any covector field $\sigma_{a}$ to the covector field $\psi \sigma_{a}$ with components

$$
\psi \sigma_{i}(P)=\partial_{i} \psi^{-1 j} \sigma_{j}\left[\psi^{-1}(P)\right] .
$$

Because a tensor is a sum of tensor products of vectors and covectors, a tensor field $T^{a \cdots b}{ }_{c \cdots d}$ is dragged to a tensor field $\psi T^{a \cdots b}{ }_{c \cdots d}^{a \cdots d}$, with components

$$
\psi T^{i \cdots j}{ }_{k \cdots l}(P)=\partial_{m} \psi^{i} \cdots \partial_{n} \psi^{j} \partial_{k} \psi^{-1 p} \cdots \partial_{l} \psi^{-1 q} T^{m \cdots n}{ }_{p \cdots q}\left[\psi^{-1}(P)\right] .
$$

Finally, the Lie derivative of any tensor is given by

$$
\mathcal{L}_{\xi} T^{a \cdots b}{ }_{c \cdots d}=-\frac{d}{d \lambda} \psi_{\lambda} T^{a \cdots b}{ }_{c \cdots d}
$$

and a calculation essentially identical to that for a vector field verifies that the definition yields Eq. (20).

\section{Integration, forms and densities}

\subsection{Introduction to integration on manifolds}

In flat space, the area of a parallelogram spanned by the vectors $\mathbf{A}, \mathbf{B}$ is $|\mathbf{A} \times \mathbf{B}|=$ $\left|\epsilon_{a b} A^{a} B^{b}\right|$; and the volume spanned the vectors $\mathbf{A}, \mathbf{B}, \mathbf{C}$ is

$|\mathbf{A} \times \mathbf{B} \cdot \mathbf{C}|=\left|\epsilon_{a b c} A^{a} B^{b} C^{c}\right|$. Similarly, in Minkowski space, requiring that the volume spanned by four orthonormal vectors $t^{\alpha}, x^{\alpha}, y^{\alpha}, z^{\alpha}$ be 1 implies that a parallelepiped $\Omega$ spanned by any four vectors $A^{\alpha}, B^{\alpha}, C^{\alpha}, D^{\alpha}$ is

$$
|\Omega|=\left|\epsilon_{\alpha \beta \gamma \delta} A^{\alpha} B^{\beta} C^{\gamma} D^{\delta}\right|
$$

The vectors are positively oriented if $\epsilon_{\alpha \beta \gamma \delta} A^{\alpha} B^{\beta} C^{\gamma} D^{\delta}>0$.

The volume of an arbitrary region $\Omega$ is obtained by adding volumes of infinitesimal parallelepipeds spanned by vectors along the coordinate axes, $e_{0}^{\alpha}, e_{1}^{\alpha}$, $e_{2}^{\alpha}, e_{3}^{\alpha}$ with lengths $\Delta x^{0}, \Delta x^{1}, \Delta x^{2}, \Delta x^{3}$

$$
\begin{aligned}
\Delta^{4} V & =\epsilon_{0123} \Delta x^{0} \Delta x^{1} \Delta x^{2} \Delta x^{3} \\
& =\frac{1}{4 !} \epsilon_{\mu \nu \sigma \tau} \Delta x^{\mu} \Delta x^{\nu} \Delta x^{\sigma} \Delta x^{\tau}(-1)^{\Pi},
\end{aligned}
$$


where $(-1)^{\Pi}=1$ when $\mu, \nu \sigma, \tau$ is an even permutation $\Pi$ of $0,1,2,3$ and $(-1)^{\Pi}=$ -1 for an odd permutation. Because $\epsilon_{\alpha \beta \gamma \delta} A^{\alpha} B^{\beta} C^{\gamma} D^{\delta}$ is a scalar, the volume of a region of flat space is given in any chart by

$$
|\Omega|=\int_{\Omega} \epsilon_{0123} d x^{0} d x^{1} d x^{2} d x^{3} \equiv \int_{\Omega} d^{4} V .
$$

The Jacobian, $\left|\frac{\partial x}{\partial x^{\prime}}\right|$, that relates the volume element in two different coordinate systems arises from the coordinate transformation of the totally antisymmetric tensor $\epsilon_{\alpha \beta \gamma \delta}$ :

$\epsilon_{0^{\prime} 1^{\prime} 2^{\prime} 3^{\prime}}=\frac{\partial x^{\mu}}{\partial x^{\prime 0}} \frac{\partial x^{\nu}}{\partial x^{\prime 1}} \frac{\partial x^{\sigma}}{\partial x^{\prime 2}} \frac{\partial x^{\tau}}{\partial x^{\prime 3}} \epsilon_{\mu \nu \sigma \tau}=\left|\frac{\partial x}{\partial x^{\prime}}\right| \epsilon_{0123}$.

A curved space is locally flat in the sense that, in a locally inertial coordinate system, the metric components are flat up to quadratic order in the coordinates. By demanding that the volumes of small regions, to first order in the length of a side, are those measured by a locally inertial observer using her local Minkowski metric, one uniquely picks out the volume element

$$
d^{4} V=\epsilon_{0123} d x^{0} d x^{1} d x^{2} d x^{3} .
$$

Because the totally antisymmetric tensor $\epsilon_{0123}$ has the value $\sqrt{|g|}$, the volume element can be written in the equivalent form ${ }^{3}$

$$
d^{4} V=\sqrt{|g|} d x^{0} d x^{1} d x^{2} d x^{3}
$$

and in n-dimensions

$$
d^{n} V=\epsilon_{1 \cdots n} d x^{1} \cdots d x^{n}=\sqrt{|g|} d x^{1} \cdots d x^{n} .
$$

In index notation, one writes

$$
d^{4} V=\epsilon_{a \cdots b} d S^{a \cdots b}
$$

and thinks of $d S^{a \cdots b}$ having components

$$
\text { “ } d S^{\mu \nu \sigma \tau}= \pm \frac{1}{4 !} d x^{\mu} d x^{\nu} d x^{\sigma} d x^{\tau} \quad \text { ”. }
$$

\footnotetext{
${ }^{3}$ The equality $\epsilon_{0123}=\sqrt{|g|}$ can be obtained as follows. In an orthonormal frame (e.g., in locally inertial coordinates), $\epsilon_{0123}$ and $\sqrt{|g|}$ each have the value 1 . Under a coordinate transformation from $x^{\mu}$ to $x^{\prime \mu}, \sqrt{|g|}$, like $\epsilon_{0123}$, is multiplied by the absolute value of the Jacobian $\left|\partial x / \partial x^{\prime}\right|$, implying that for any positively oriented coordinate system, $\sqrt{|g|}=\epsilon_{0123}$.
} 
The integral,

$$
\int_{\Omega} f d^{n} V=\int_{\Omega} f \epsilon_{1 \cdots n} d x^{1} \cdots d x^{n}=\int_{\Omega} f \sqrt{|g|} d x^{1} \cdots d x^{n}
$$

over a region $\Omega$ is is well-defined (that is, its value is independent of the choice of coordinates), because, under a change of coordinates, the integrand on the right side is multiplied by the Jacobian $\left|\frac{\partial x}{\partial x^{\prime}}\right|$.

\subsection{Forms and densities}

The tensor $\epsilon_{a \cdots b}$ and the quantity $\sqrt{|g|}$ that appear in the alternative ways of writing an integral are, respectively, an example of a form and a scalar density. As we will see, there is a duality between forms and densities that underlies a equivalence between Stokes's theorem and Gauss's theorem. We define forms and densities, present the duality that relates them, and go on to the corresponding duality relating the integral theorems.

Forms.

Definition. A $p$-form $\sigma_{a \cdots b}$ is an antisymmetric, covariant tensor with $p$ indices. In particular, a scalar $f$ is a 0 -form, a covariant vector $A_{a}$ is a 1 -form, and an antisymmetric 2-index tensor $F_{a b}$ is a 2-form.

Definition. The exterior derivative $d \sigma$ of a $p$-form $\sigma$ is the $p+1$ form

$$
(d \sigma)_{a b \cdots c}=(p+1) \nabla_{[a} \sigma_{b \ldots c]} .
$$

The factor $p+1$ is the number of independent ways of distributing the $p+1$ indices between $\nabla$ and $\sigma$. The antisymmetry implies that $d \sigma$ is independent of the derivative operator; in any chart it has components

$$
(d \sigma)_{i j \cdots k}=(p+1) \partial_{[i} \sigma_{j \ldots k]}
$$

Antisymmetry and the commutativity of partial derivatives imply for any form $\sigma$

$$
d^{2} \sigma=0
$$

Lie derivatives and exterior derivatives commute,

$$
\mathcal{L}_{\mathbf{v}} d \sigma=d \mathcal{L}_{\mathbf{v}} \sigma
$$


and the two derivatives satisfy the Cartan identity,

$$
\mathcal{L}_{\mathbf{v}} \sigma=v \cdot(d \sigma)+d(v \cdot \sigma), \text { where }(v \cdot \sigma)_{a \cdots b}:=v^{c} \sigma_{c a \cdots b} .
$$

Each relation can be proved by induction on $p$ (the number of indices of $\sigma$ ). For an $n$-form in $n$ dimensions, the second relation can be written as

$$
\mathcal{L}_{\mathbf{v}} \sigma_{a \cdots b}=\nabla_{c}\left(\sigma_{a \cdots b} v^{c}\right)
$$

with special case

$$
\mathcal{L}_{\mathbf{v}} \epsilon_{a \cdots b}=\nabla_{c}\left(\epsilon_{a \cdots b} v^{c}\right)=\epsilon_{a \cdots b} \nabla_{c} v^{c} .
$$

In $n$-dimensions, any nonzero $n$-form is functionally proportional to any other, because each has only one independent component. In particular, because any $n$ form $\sigma$ is given by $\sigma_{a \cdots b}=f \epsilon_{a \cdots b}$ for some scalar $f$, the integral of an $n$-form is well-defined, given in any coordinate system by

$$
\int_{\Omega} \sigma_{a \cdots b} d S^{a \cdots b}=\int \sigma_{1 \cdots n} d x^{1} \cdots d x^{n}
$$

(Again the integral is well defined because a change of coordinates multiplies the value of a $p$-form by the Jacobian of the transformation.)

\section{Densities.}

A scalar density $\mathfrak{f}$ (of weight 1 ), by definition, transforms under a change of coordinates in the same way as one component of an $n$-form in $n$ dimensions: $\mathfrak{f} \rightarrow\left|\frac{\partial x}{\partial x^{\prime}}\right| \mathfrak{f}$. Just as one can write any $n$-form as a multiple of $\epsilon_{a \cdots b}$ (once one is given a metric), one can write any scalar density as a scalar multiple of $\sqrt{|g|}$ :

$$
\mathfrak{f}=f \sqrt{|g|},
$$

for some scalar $f$ (namely $\mathfrak{f} / \sqrt{|g|}$ ). One can analogously introduce vector and tensor densities by transformation laws that differ from those of vectors and tensors by the Jacobian of the transformation: The change of the components of a vector density under a coordinate transformation is given by $\mathfrak{j}^{i} \rightarrow\left|\frac{\partial x}{\partial x^{\prime}}\right| \frac{\partial x^{\prime i}}{\partial x^{k}} \mathfrak{j}^{k}$. Again one can write any vector density in the form $j^{a}=j^{a} \sqrt{|g|}$, with $j^{a}$ a vector field.

The Lie derivative of a scalar or tensor density can be deduced from this fact as follows (a metric-free derivation uses the geometric definition of Lie derivative 
and the fact that the action of a diffeo on a density differs from its action on a tensor by the inverse Jacobian of the diffeo): We have

$$
\mathcal{L}_{\mathbf{v}} \sqrt{|g|}=\frac{\partial \sqrt{|g|}}{\partial g_{a b}} \mathcal{L}_{\mathbf{v}} g_{a b}=\frac{1}{2} \frac{1}{\sqrt{|g|}} \frac{\partial g}{\partial g_{a b}} \mathcal{L}_{\mathbf{v}} g_{a b}
$$

Now the coefficient of the component $g_{i j}$ in the determinant $g$ is the minor $\Delta^{i j}$ of $g_{i j}$, and the inverse metric is given by $g^{i j}=\frac{\Delta^{i j}}{g}$. Thus $\frac{\partial g}{\partial g_{i j}}=\Delta^{i j}=g g^{i j}$, and we have

$$
\mathcal{L}_{\mathbf{v}} \sqrt{|g|}=\frac{1}{2} \sqrt{|g|} g^{a b} \mathcal{L}_{\mathbf{v}} g_{a b}=\sqrt{|g|} \nabla_{a} v^{a}=\nabla_{a}\left(\sqrt{|g|} v^{a}\right) .
$$

Finally, Eqs. (2) and (39) imply that the Lie derivative of a general scalar density $\mathfrak{f}$ is

$$
\mathcal{L}_{\mathbf{v}} \mathfrak{f}=\nabla_{a}\left(\mathfrak{f} v^{a}\right)
$$

Duality. A duality between $p$-forms $\sigma_{a_{1} \cdots a_{p}}$ and antisymmetric tensor densities $\mathcal{A}^{a_{1} \cdots a_{q}}$, with $q=n-p$ indices ( $n$ the dimension of the space) is given by

$$
\mathcal{A}^{a_{1} \cdots a_{q}}=\frac{1}{p !} \sqrt{|g|} \epsilon^{a_{1} \cdots a_{q} b_{1} \cdots b_{p}} \sigma_{b_{1} \cdots b_{p}} .
$$

The inverse relation is $\sigma_{a_{1} \cdots a_{p}}= \pm \frac{1}{q !}|g|^{-1 / 2} \epsilon_{a_{1} \cdots a_{p} b_{1} \cdots b_{q}} \mathcal{A}^{b_{1} \cdots b_{q}}$, where the sign is positive for a positive definite metric, negative for a metric with Lorentz signature.

The divergence $\nabla \cdot \mathcal{A}$, of an antisymmetric density,

$$
(\nabla \cdot \mathcal{A})^{b \cdots c}:=\nabla_{c} \mathcal{A}^{c a \cdots b}
$$

is dual to the exterior derivative $d \sigma$ of the form $\sigma$ dual to $\mathcal{A}$, and it is independent of the choice of $\nabla$, having components

$$
\nabla_{k} \mathcal{A}^{k i \cdots j}=\partial_{k} \mathcal{A}^{k i \cdots j}
$$

In particular, the equation for the divergence of a vector density $\mathcal{A}^{a}$,

$$
\nabla_{a} \mathcal{A}^{a}=\partial_{i} \mathcal{A}^{i}
$$

is equivalent (once one has a metric) to the familiar relation $\nabla_{a} A^{a}=\frac{1}{\sqrt{|g|}} \partial_{i}\left(\sqrt{|g|} A^{i}\right)$. 
The relations (33), (34), and (35) are dual to the relations

$$
\begin{aligned}
\nabla \cdot(\nabla \cdot \mathcal{A}) & =0 \\
\mathcal{L}_{\mathbf{v}} \nabla \cdot \mathcal{A} & =\nabla \cdot \mathcal{L}_{\mathbf{v}} \mathcal{A} \\
\mathcal{L}_{\mathbf{v}} \mathcal{A} & =\nabla \cdot(v \wedge \mathcal{A})+v \wedge \nabla \cdot \mathcal{A}
\end{aligned}
$$

where $(v \wedge \mathcal{A})^{a b \cdots c}:=(q+1) v^{[a} \mathcal{A}^{b \cdots c]}$.

On an $n$-dimensional manifold, integrals are naturally defined for $n$-forms or for scalar densities, which can be said to be dual to their corresponding $n$-forms. That is, the integrals

$$
\int \omega_{a \cdots b} d S^{a \cdots b}=\int \omega_{1 \cdots n} d x^{1} \cdots d x^{n} \quad \text { and } \quad \int \mathfrak{f} d x^{1} \cdots d x^{n}
$$

are well defined because under a change of coordinates, each integrand is multiplied by the Jacobian of the transformation. The mathematical literature adopts an index-free notation in which the integral of an $n$-form $\omega$ over an $n$-dimensional region $\Omega$ is written $\int_{\Omega} \omega$.

\subsection{Diffeomorphism invariance}

The usual invariance of an integral under a coordinate transformation has as its active equivalent the invariance of an integral under a diffeo. Let $\omega_{a \ldots b}$ be an $n$ form on an $n$-dimensional volume $V$. With $\psi(V)$ the image of $V$ and $\psi \omega_{a \ldots b}$ the dragged $n$-form, the invariance relation is

$$
\int_{\psi(V)} \psi \omega=\int_{V} \omega .
$$

As in the case of coordinate transformations, invariance under diffeos follows from the fact that the components of $\omega_{a \ldots b}$ change by a Jacobian. Intuitively, the invariance follows from the physical equivalence of diffeo-related tensors on diffeo-related domains.

A corollary for a family of diffeos, $\psi_{\lambda}$, is used to obtain the relation between conservation of vorticity and conservation of circulation:

Let $\xi^{a}$ be the generator of the family $\psi_{\lambda}$. Then

$$
\frac{d}{d \lambda} \int_{\psi_{\lambda}(V)} \omega=\int_{V} \mathcal{L}_{\xi} \omega .
$$


The proof is immediate from Eq. (26), starting from Eq. (49) in the form

$\int_{\psi_{\lambda}(V)} \omega=\int_{V} \psi_{-\lambda} \omega: \quad \frac{d}{d \lambda} \int_{\psi_{\lambda}(V)} \omega=\int_{V} \frac{d}{d \lambda} \psi_{-\lambda} \omega=\int_{V} \mathcal{L}_{\xi} \omega$.

In particular, for $\psi_{\tau}$ the family of diffeos describing the flow of a fluid with 4velocity $u^{\alpha}$, we have

$$
\frac{d}{d \tau} \int_{c_{\tau}} h u_{\alpha} d l^{\alpha}=\int_{c} \mathcal{L}_{\mathbf{u}}\left(h u_{\alpha}\right) d l^{\alpha}
$$

where $c$ is a closed curve in the fluid and $c_{\tau}=\psi_{\tau}(c)$.

\section{Gauss's theorem and Stokes's theorem}

To understand the relation between differential and integral conservation laws for relativistic stars and black holes, one needs the generalization of Stokes's theorem and Gauss's theorem to manifolds. It is helpful to see quickly how these generalizations go before we move to a more formal presentation. The simplest version of Stokes's theorem is its 2-dimensional form, namely Green's theorem:

$$
\int_{S}\left(\partial_{x} A_{y}-\partial_{y} A_{x}\right) d x d y=\int_{c}\left(A_{x} d x+A_{y} d y\right),
$$

where $c$ is a curve bounding the 2 -surface $S$. The theorem involves the integral over a 2-surface of the antisymmetric tensor $\nabla_{a} A_{b}-\nabla_{b} A_{a}$. In three dimensions, the tensor is dual to the curl of $\mathbf{A}:(\nabla \times \mathbf{A})^{a}=\epsilon^{a b c} \nabla_{b} A_{c}$; and Stokes's generalization of Green's theorem can be written in either the form

$$
\int_{S}(\boldsymbol{\nabla} \times \boldsymbol{A}) \cdot d \mathbf{S}=\int_{c} \mathbf{A} \cdot \mathbf{d} \mathbf{l}
$$

or in terms of the antisymmetric tensor $\nabla_{a} A_{b}-\nabla_{b} A_{a}$

$$
\int_{S}\left(\nabla_{a} A_{b}-\nabla_{b} A_{a}\right) d S^{a b}=\int_{c} A_{a} d l^{a}
$$

where, for an antisymmetric tensor $F_{a b}, F_{a b} d S^{a b}$ means $F_{12} d x^{1} d x^{2}+F_{23} d x^{2} d x^{3}+$ $F_{31} d x^{3} d x^{1}$. Written in this form, the theorem is already correct in a curved spacetime. The reason is that the antisymmetric derivative $\nabla_{a} A_{b}-\nabla_{b} A_{a}$ has in curved space the same form it has in flat space: As we have seen, its components in any coordinate system are just $\partial_{i} A_{j}-\partial_{j} A_{i}$. 
As a result, the flat-space proof of Green's theorem and Stokes's theorem, based on the Fundamental Theorem of calculus $\left(\int_{a}^{b} f^{\prime}(x) d x=f(b)-f(a)\right.$ ), holds in curved space as well: Let $S$ be a coordinate square in a surface of constant $x^{3}$. Then

$$
\int_{S}\left(\partial_{1} A_{2}-\partial_{2} A_{1}\right) d x^{1} d x^{2}=\int_{c}\left(A_{1} d x^{1}+A_{2} d x^{2}\right),
$$

with the boundary of the square traversed counterclockwise as seen from above the square.

Gauss's theorem,

$$
\int_{V} \nabla_{a} A^{a} d^{3} x=\int_{S} A^{a} d S_{a}
$$

with $S$ a surface bounding the volume $V$ again has a simple generalization to curved space. Although the divergence $\nabla_{a} A^{a}$ does not have components $\partial_{i} A^{i}$, the divergence of $\mathcal{A}^{a}:=A^{a} \sqrt{g}$ does: $\nabla_{a} \mathcal{A}^{a}=\partial_{i} \mathcal{A}^{i}$. Again the flat-space proof of Gauss's theorem follows from an integration over a coordinate cube using the fundamental theorem of calculus for the integral over each coordinate; and again the integration over a coordinate cube has the identical form in curved space. If $V$ is a coordinate cube

$$
\int_{V} \partial_{i} \mathcal{A}^{i} d x^{1} d x^{2} d x^{3}=\int_{S}\left(\mathcal{A}^{1} d x^{2} d x^{3}+\mathcal{A}^{2} d x^{1} d x^{3}+\mathcal{A}^{3} d x^{1} d x^{2}\right)
$$

and any volume is approximated by an arbitrarily fine division into coordinate cubes. 


\subsection{Gauss's theorem (divergence theorem)}

In $\mathbb{R}^{n}$, in Cartesian coordinates, the integral of a divergence over an $n$-cube can be expressed as a surface integral after an integration by parts:

$$
\begin{aligned}
\int_{V} \partial_{i} A^{i} d^{n} x= & \int_{V} \partial_{1} A^{1} d x^{1} d x^{2} \cdots d x^{n}+\cdots+\int_{V} \partial_{n} A^{n} d x^{n} d x^{1} \cdots d x^{n-1} \\
= & \int_{\partial_{1+V}} A^{1} d x^{2} \cdots d x^{n}-\int_{\partial_{1-V}} A^{1} d x^{2} \cdots d x^{n}+\cdots \\
& +\int_{\partial_{n+V}} A^{n} d x^{1} \cdots d x^{n-1}-\int_{\partial_{n-V}} A^{n} d x^{1} \cdots d x^{n-1} \\
= & \int_{\partial V} A^{i} d S_{i} \quad(\partial V \text { means the boundary of } V) \\
& \text { where } d S_{i}= \pm \epsilon_{i j \cdots k} d x^{j} \cdots d x^{k} \frac{1}{(n-1) !}, \text { with } \\
& d S_{1}=+d x^{2} \cdots d x^{n} \text { for } x^{1} \text { increasing outward, } \\
& d S_{1}=-d x^{2} \cdots d x^{n}, \text { for } x^{1} \text { increasing inward. }
\end{aligned}
$$

More generally the integral over any volume $V$ in $\mathbb{R}^{n}$ of a divergence is related to a surface integral by

$$
\int_{V} \partial_{i} A^{i} d^{n} V=\int_{\partial V} A^{i} d S_{i}=\int_{\partial V} A^{i} n_{i} d S
$$

where $n_{i}$ is the unit outward normal to $S$ (along the gradient of an outwardly increasing scalar) and $d S$ the area element of $S$. In curved space the analogous result follows immediately from the form of the divergence of a vector density:

$$
\int_{\Omega} \nabla_{a} A^{a} d^{n} V=\int_{\Omega} \partial_{i}\left(\sqrt{|g|} A^{i}\right) d^{n} x .
$$

To generalize Gauss's theorem to an $n$-dimensional manifold $M$ with metric $g_{a b}$, let $\Omega$ be an $n$-dimensional submanifold with smooth boundary $\partial \Omega$. Define a surface element $d S_{a}$ on $\partial \Omega$ by requiring that, in any chart $x^{1}, \ldots, x^{n}$ for which $x^{1}$ is constant on $\partial \Omega$ and increasing outward,

$$
d S_{i}=\nabla_{i} x^{1} \sqrt{|g|} d x^{2} \cdots d x^{n}=\delta_{i}^{1} \sqrt{|g|} d x^{2} \cdots d x^{n} .
$$

This definition involves no choice of orientation (in fact $\Omega$ need not be orientable, as long as the outward normal to $\partial \Omega$ is well-defined.) 
Gauss's Theorem in Curved Space. For any smooth vector field $A^{a}$ on $M$,

$$
\int_{\Omega} \nabla_{a} A^{a} d^{n} V=\int_{\partial \Omega} A^{a} d S_{a} .
$$

Sketch of proof. When $\Omega$ is a coordinate cube the steps in Eq. (54) go through as written, with $A^{i}$ replaced by $\mathcal{A}^{i}=A^{i} \sqrt{|g|}$ :

$$
\begin{aligned}
\int_{\Omega} \partial_{i} \mathcal{A}^{i} d^{n} x= & \int_{\partial_{1+V} V} \mathcal{A}^{1} d x^{2} \cdots d x^{n}-\int_{\partial_{1-V} V} \mathcal{A}^{1} d x^{2} \cdots d x^{n}+\cdots \\
& +\int_{\partial_{n+V} V} \mathcal{A}^{n} d x^{1} \cdots d x^{n-1}-\int_{\partial_{n-V}} \mathcal{A}^{n} d x^{1} \cdots d x^{n-1} \\
= & \int_{\partial \Omega} A^{i} d S_{i} .
\end{aligned}
$$

Decomposing the volume of integration into a set of coordinate cubes and taking the limit as the size of each cube shrinks to zero, yields

$$
\int_{\Omega} \nabla_{a} A^{a} d^{n} V=\int_{\partial \Omega} A^{a} d S_{a} ;
$$

as usual, surface terms from cubes that share a surface cancel, because the outward normal to one cube is the inward normal to the adjacent cube.

This form is correct for a region in a space with a metric, independent of the signature of the metric. When $\partial \Omega$ has a unit outward normal $n_{a}$ (along the gradient of a scalar that increases outward), one can write $d S_{a}$ in the form $d S_{a}=n_{a} d S$. In this case,

$$
\int \nabla_{a} A^{a} d^{n} V=\int A^{a} n_{a} d S .
$$

Example: The integral form of baryon mass conservation $\nabla_{\alpha}\left(\rho u^{\alpha}\right)=0$ is

$$
\begin{aligned}
0 & =\int_{\Omega} \nabla_{\alpha}\left(\rho u^{\alpha}\right) d^{4} V=\int_{\partial \Omega} \rho u^{\alpha} d S_{\alpha} \\
& =\int_{V_{2}} \rho u^{\alpha} d S_{\alpha}-\left|\int_{V_{1}} \rho u^{\alpha} d S_{\alpha}\right| .
\end{aligned}
$$

Here the fluid is taken to have finite spatial extent, and the spacetime region $\Omega$ is bounded by the initial and final spacelike hypersurfaces $V_{1}$ and $V_{2}$. In a coordinate 
system for which $V_{1}$ and $V_{2}$ are surfaces of constant $t$, with $t$ increasing to the future, we have $d S_{\mu}=\nabla_{\mu} t \sqrt{|g|} d^{3} x=\delta_{\mu}^{t} \sqrt{|g|} d^{3} x$ on $V_{2}, d S_{\mu}=-\delta_{\mu}^{t} \sqrt{|g|} d^{3} x$ on $V_{1}$, and

$$
\int_{\Omega} \nabla_{\alpha}\left(\rho u^{\alpha}\right) d^{4} V=\int_{V_{2}} \rho u^{t} \sqrt{|g|} d^{3} x-\int_{V_{1}} \rho u^{t} \sqrt{|g|} d^{3} x .
$$

If, on a slicing of spacetime one chooses on each hypersurface $V$ a surface element $d S_{\alpha}$ along $+\nabla_{\alpha} t$, the conservation law is then

$$
M_{0}=\int_{V} \rho u^{\alpha} d S_{\alpha}=\text { constant }
$$

Note that the fact that one can write the conserved quantity associated with a current $j^{\alpha}$ in the form,

$$
\int_{V} j^{\alpha} d S_{\alpha}=\int_{V} j^{t} \sqrt{|g|} d^{3} x
$$

means that there is no need to introduce $n_{\alpha}$ and $\sqrt{{ }^{3} g}$ to evaluate the integral. This fact is essential if one is evaluating an integral $\int j^{\alpha} d S_{\alpha}$ over a null surface, where there is no unit normal. The flux of energy or of baryons across the horizon of a Schwarzschild black hole, for example, can be computed in Eddington-Finkelstein or Kruskal coordinates: In ingoing Eddington-Finkelstein coordinates $v, r, \theta, \phi$, the horizon is a surface of constant $r$, and we have

$$
\int j^{\alpha} d S_{\alpha}=\int j^{r} \sqrt{|g|} d v d \theta d \phi
$$

\subsubsection{Generalized divergence theorem}

The key to Gauss's theorem is the fact that the divergence of a vector density has the form $\partial_{i}\left(\sqrt{|g|} A^{i}\right)$ or $\partial_{i} \mathcal{A}^{i}$. This is true of any $q$-index antisymmetric tensor density, $\mathcal{A}^{a \cdots b}=A^{a \cdots b} \sqrt{|g|}$, and an analogous theorem holds. Because the text uses only the case of a two-index antisymmetric tensor, the electromagnetic field $F^{\alpha \beta}$, and because the way one extends the proof will be clear, we will give the generalization in detail for this case. The theorem now relates an integral over an $n-1$-dimensional submanifold $S$ of $M$ to an integral over its $n-2$-dimensional boundary $\partial S$ :

$$
\int_{S} \nabla_{b} A^{a b} d S_{a}=\int_{\partial S} A^{a b} d S_{a b}
$$


where the sign of $d S_{a}$ and the meaning of $d S_{a b}$ are defined as follows.

Let $x^{1}, \ldots, x^{n}$ be a positively oriented chart on a subset of $M$ for which $x^{1}$ is constant on $S$, and with $x^{2}$ constant on $\partial S$ and increasing outward. Choose the sign of $d S_{a}$ by $d S_{a}=\epsilon_{a b \cdots c} d S^{b \cdots c}$ or, equivalently, by requiring $d S_{a}=\nabla_{a} x^{1} \sqrt{|g|} d x^{2} \cdots d x^{n}$. The volume element $d S_{a b}$ is similarly chosen to satisfy, in our oriented chart,

$$
d S_{a b}=\nabla_{[a} x^{1} \nabla_{b]} x^{2} \sqrt{|g|} d x^{3} \cdots d x^{n} .
$$

Then in the coordinates' domain,

$$
\int_{S} \nabla_{b} A^{a b} d S_{a}=\int_{S} \nabla_{b}\left(A^{a b} \nabla_{a} x^{1} \sqrt{|g|}\right) d x^{2} \cdots d x^{n} .
$$

But the last integrand is just the divergence of the vector density $\widetilde{\mathcal{A}}^{b}=A^{a b} \nabla_{a} x^{1} \sqrt{|g|}$, and we have already proved Gauss's law for this case:

$$
\int_{S} \nabla_{b} \tilde{A}^{b} \sqrt{|g|} d x^{2} \cdots d x^{n}=\int_{\partial S} \tilde{A}^{b} d \tilde{S}_{b}
$$

where, in each chart, $d \tilde{S}_{b}=\nabla_{b} x^{2} \sqrt{|g|} d x^{3} \cdots d x^{n}$. Finally, $\tilde{A}^{b} d S_{b}=A^{a b} d S_{a b}$, whence Eq. (65) is identical to the generalized divergence theorem, Eq. (62).

Example (Electric charge). Let $V$ be a ball containing a charge Q. The 4-dimensional form of Gauss's law relates the charge $Q=\int_{V} j^{\alpha} d S_{\alpha}$ in $V$ to the electric flux $\int F^{\alpha \beta} d S_{\alpha \beta}$ through the 2-dimensional surface of $V$ :

$$
Q=\int_{V} j^{\alpha} d S_{\alpha}=\frac{1}{4 \pi} \int_{V} \nabla_{\beta} F^{\alpha \beta} d S_{\alpha}=\frac{1}{4 \pi} \int_{\partial V} F^{\alpha \beta} d S_{\alpha \beta}
$$

Pick positively oriented coordinates $t, r, \theta, \phi$ for which $V$ is a $t=$ constant surface and $\partial V$ an $r=$ constant surface. Then

$$
Q=\frac{1}{4 \pi} \int F^{t r} \sqrt{|g|} d \theta d \phi
$$

Flat space:

$$
F^{t r}=E^{r}=\frac{Q}{r^{2}} \Longrightarrow \quad \int_{V} j^{\alpha} d S_{\alpha}=\frac{1}{4 \pi} \int\left(\frac{Q}{r^{2}}\right)\left(r^{2} \sin \theta\right) d \theta d \phi=\frac{Q}{4 \pi} \int d \Omega=Q
$$

For a $q$-index antisymmetric tensor $A^{a \cdots b c}$, the generalized divergence theorem takes the form $\int_{S} \nabla_{c} A^{a \cdots b c} d S_{a \cdots b}=\int_{\partial S} A^{a \cdots b c} d S_{a \cdots b c}$. 


\subsection{Stokes's theorem}

The divergence $\nabla_{a} A^{a}$ of a vector on an $n$-dimensional manifold is dual to the exterior derivative $(d \omega)_{a b \cdots c}=n \nabla_{[a} \omega_{b \cdots c]}$ of the $(n-1)$-form $\omega$ dual to $A^{a}$. That is, with

$$
\omega_{b \cdots c}:=A^{a} \epsilon_{a b \cdots c}
$$

we have

$$
\epsilon_{a b \cdots c} \nabla_{d} A^{d}=(d \omega)_{a b \cdots c} .
$$

Because both sides of the equation are $n$-forms, one need only check one component: $n \nabla_{[1} \omega_{2 \cdots n]}=\nabla_{1}\left(A^{d} \epsilon_{d 2 \cdots n}\right)-\nabla_{2}\left(A^{d} \epsilon_{d 13 \cdots n}\right)-\cdots-\nabla_{n}\left(A^{d} \epsilon_{d 2 \cdots n-11}\right)=$ $\epsilon_{12 \cdots n} \nabla_{d} A^{d}$. The corresponding dual of the generalized divergence theorem above is called

Stokes's Theorem. Let $\omega$ be an $n-1$-form on an $n$-dimensional manifold $S$ with boundary $\partial S$. Then

$$
\int_{S} d \omega=\int_{\partial S} \omega
$$

In index notation,

$$
\int_{S}(d \omega)_{a b \cdots c} d S^{a b \cdots c}=\int_{\partial S} \omega_{b \cdots c} d S^{b \cdots c}
$$

The theorem implicitly assumes an orientation for $\partial S$ obtained from that of $S$ by requiring that, if $x^{1}, \cdots, x^{n}$ is a positively oriented chart on $S$ with $\partial S$ a surface of constant $x^{1}$ and $x^{1}$ increasing outward, then $x^{2}, \cdots, x^{n}$ is a positively oriented chart for $\partial S$.

Proof. This dual of Gauss's theorem follows quickly from Eq. (55), in the form

$$
\int_{S} \nabla_{d} A^{d} \epsilon_{a b \cdots c} d S^{a b \cdots c}=\int_{\partial S} A^{a} d S_{a} .
$$

Define $A^{a}$ by Eq. (68), and note that, with the orientation chosen above, $d S_{a}=$ $\epsilon_{a b \cdots c} d S^{b \cdots c}$. Then

$$
\int_{S} \nabla_{d} A^{d} \epsilon_{a b \cdots c} d S^{a b \cdots c}=\int_{\partial S} A^{a} \epsilon_{a b \cdots c} d S^{b \cdots c},
$$

and, from Eqs. (68) and (69), the result follows:

$$
\int_{S}(d \omega)_{a b \cdots c} d S^{a b \cdots c}=\int_{\partial S} \omega_{b \cdots c} d S^{b \cdots c} .
$$


Example: Stokes's theorem in three dimensions.

Let $A_{a}$ be a 3-vector. $(d A)_{a b}=\nabla_{a} A_{b}-\nabla_{b} A_{a}$

$$
\int_{S}\left(\nabla_{a} A_{b}-\nabla_{b} A_{a}\right) d S^{a b}=\int_{c} A_{a} d l^{a},
$$

$c$ the curve bounding $S$. As noted at the beginning of this section, this is equivalent to the usual form of Stokes's theorem in vector calculus:

$\int_{S} \vec{\nabla} \times \vec{A} \cdot d \vec{S}=\int_{S}\left(\epsilon^{a b c} \nabla_{b} A_{c}\right) \epsilon_{a d e} d S^{d e}=\int_{S}\left(\nabla_{a} A_{b}-\nabla_{b} A_{a}\right) d S^{a b}=\int_{c} \vec{A} \cdot d \vec{l}$.

\section{References}

[1] E. A. Coddington and N. Levinson. Theory of Ordinary Differential Equations. McGraw-Hill, New York, 1955. 